\title{
GMR
}

\section{Expression levels of survivin, Bcl-2, and KAl1 proteins in cervical cancer and their correlation with metastasis}

\author{
X.L. Zhou' and M. Wang ${ }^{2}$ \\ 1'Department of Gynecology, Liaocheng Second Hospital, Taishan Medical College, \\ Linqing, Shandong Province, China \\ 2Department of Pathology, Liaocheng Second Hospital, Taishan Medical College, \\ Linqing, Shandong Province, China \\ Corresponding author: X.L. Zhou \\ E-mail: xinlingShandong96@163.com \\ Genet. Mol. Res. 14 (4): 17059-17067 (2015) \\ Received August 31, 2015 \\ Accepted October 30, 2015 \\ Published December 16, 2015 \\ DOI http://dx.doi.org/10.4238/2015.December.16.6
}

\begin{abstract}
Cervical cancer is associated with abnormal expression of multiple genes. Survivin and $\mathrm{Bcl}-2$ proteins are apoptosis inhibitors. The tumor suppressor gene $C D 82$, which encodes the protein KAl1, is downregulated in cervical cancer, and is associated with differentiation degree. We investigated the expression levels of three proteins and their correlation with metastasis in cervical cancer by comparing them in different cervical lesions. Immunohistochemistry was used to detect their three protein expression levels in the normal cervix, chronic cervicitis, cervical intraepithelial neoplasia (CIN) lesions, and cervical cancer. The relationships between the protein expression levels and tumor type, clinical stage, tissue differentiation, invasion, and metastasis were analyzed. Survivin and Bcl-2 expression levels in cervical cancer were significantly higher than in the normal cervix, chronic cervicitis, or $\mathrm{CIN}(\mathrm{P}<$ 0.05). KAl1 expression was markedly lower in cervical cancer than in the normal cervix, chronic cervicitis, or $\mathrm{CIN}(P<0.05)$. There was no statistical difference between the expression levels of the three proteins in CIN and
\end{abstract}


chronic cervicitis, but there were differences in expression between CIN and normal cervical tissues $(P<0.05)$. Bcl-2 and survivin levels were positively correlated while KAI1 expression was negatively correlated with clinical stage. Survivin and KAl1 expression levels were associated with lymph node metastasis $(P<0.05)$, and KAl1 expression was positively related with differentiation degree $(P<0.05)$. Survivin, $\mathrm{Bcl}-2$, and KAl1 are metastasis-related factors in cervical cancer. Overexpression of survivin and $\mathrm{Bcl}-2$, and low expression of KAl1 promotes cervical cancer progress and metastasis.

Key words: Cervical cancer; Bcl-2, Survivin; KAl1; Metastasis

\section{INTRODUCTION}

Cervical cancer is a common malignant tumor of the female reproductive organs. In recent years, the incidence of cervical cancer has increased annually as a result of environmental and life stresses. Furthermore, there is a trend toward earlier onset. Distant metastasis is a major cause of death. Patients with advanced cervical cancer present a poor prognosis. Early diagnosis and treatment represent the key to increasing cervical cancer survival rates and improving quality of life in patients. Investigations have focused on the mechanisms associated with cervical cancer invasion and metastasis, which are important for the improvement of patient prognoses (Ozden et al., 2014; Winer et al., 2015) Tumorigenesis is associated with multiple genes and the interaction between several factors. The pathological process of cervical cancer is related to apoptosis suppression, which is regulated by tumor-suppressor gene inactivation, oncogene activation or mutation, and anti-apoptosis gene overexpression (Ouyang et al., 2008a,b). Survivin and Bcl-2 are anti-apoptosis proteins (Adefolaju et al., 2014; Liu et al., 2014). BCL2, which has long been recognized as an apoptosis regulation gene, has received in-depth study, and its rate of expression increases in cervical cancer tissue. The BIRC5 gene, which encodes the protein survivin, strongly inhibits apoptosis, and its expression level is elevated following a decrease in cancer differentiation. The tumor suppressor geneCD82, which encodes the protein KAl1, can suppress metastasis in a variety of tumors, such as lung, prostate, and colon cancers. However, the relationship between $\mathrm{KAI} 1$ and cervical cancer is controversial. KAI1 is a cell surface glycoprotein that participates in cell adhesion regulation and impedes tumor cell separation, thereby inhibiting tumor metastasis. CD82 gene expression is downregulated in the early stages of cervical cancer (Wang et al., 2001; Singh et al., 2004). In this study, we investigated the relationship between the expression levels of three proteins and the various clinicopathologic characteristics of cervical cancer by comparing them at different stages of the cancer: normal tissue, chronic cervicitis, cervical intraepithelial neoplasia (CIN) lesions, and cervical cancer.

\section{MATERIAL AND METHODS}

\section{Clinical information}

Tissue specimens were selected from 25 cases of normal cervical tissue, 25 cases of chronic cervicitis, 30 cases of $\mathrm{CIN}$, and 45 cases of cervical cancer ( 41 squamous cell carcinomas 
and 4 adenocarcinomas). All hematoxylin and eosin (H\&E)-stained slices were confirmed by two pathologists. The mean age of the 45 cervical cancer patients was $36.5 \pm 4.2(26-51)$ years. There were 14 cases with high differentiation, 28 cases with moderate differentiation, and 3 cases with poor differentiation. Furthermore, 4 cases were at stage I, 38 cases were at stage Ib-lla, and 3 cases were at stage IIb-III. Lymph node metastases were present in 29 cases and absent in 16 cases. No patients received chemotherapy or radiotherapy before surgery. The patients who did not undergo surgery were diagnosed by magnetic resonance imaging, while those who did were diagnosed by pathology.

The mean age of the 30 patients with CIN was $37.1 \pm 3.4$ (25-50) years. There were 10 cases at CIN level I, 12 cases at CIN level II, and 8 cases at CIN level III. The mean age of the 25 normal controls was $36.8 \pm 3.6(25-52)$ years, and the patients with chronic cervicitis were $37.3 \pm$ $3.33(26-52)$ years old. There was no significant difference among the different groups $(P>0.05)$ (Tables 1 and 2).

\begin{tabular}{|c|c|c|c|c|c|c|c|}
\hline \multirow[t]{2}{*}{ Group } & \multirow[t]{2}{*}{$\mathrm{N}$} & \multirow[t]{2}{*}{ Mean age $(y)$} & \multicolumn{3}{|c|}{ Differentiation (No.) } & \multicolumn{2}{|c|}{ Lymph node metastasis (No.) } \\
\hline & & & Good & Moderate & Poor & With & Without \\
\hline Normal control & 25 & $36.8 \pm 3.6$ & - & - & - & - & \\
\hline Chronic cervicitis & 25 & $37.3 \pm 3.3$ & - & - & - & - & \\
\hline CIN & 30 & $37.1 \pm 3.4$ & - & & - & - & \\
\hline Cervical cancer & 45 & $36.5 \pm 4.2$ & 14 & 28 & 3 & 29 & 1 \\
\hline
\end{tabular}

$\mathrm{CIN}=$ cervical intraepithelial neoplasia.

Table 2. Clinical staging of patients with different cervical lesions.
\begin{tabular}{lcccc}
\hline Group & N & & Clinical staging (No.) & III \\
\cline { 2 - 5 } & & I & - & - \\
Normal control & 25 & - & - & - \\
Chronic cervicitis & 25 & - & 12 & 8 \\
CIN & 30 & 10 & 38 & 3 \\
Cervical cancer & 45 & 4 &
\end{tabular}

$\mathrm{CIN}=$ cervical intraepithelial neoplasia.

The study protocol was approved by the Research Ethics Committee of our hospital, and all patients gave their informed consent before study commencement.

\section{Methods}

Immunohistochemistry was used to determine survivin, Bcl-2, and KAl1 protein expression levels in the different cervical tissues according to the manufacturer instructions supplied by the Beijing Zhongshan Biotechnology Co., Ltd (Beijing, China). Cervical tissue specimens were continuously resected into $4-\mu \mathrm{m}$ sections and treated with Bcl-2 monoclonal antibody (1:50), survivin polyclonal antibody (1:100), or KAl1 polyclonal antibody (1:200). The slices were observed following paraffin dewaxing, microwave antigen repair, 3,3'-diaminobenzidine staining, hematoxylin re-dyeing, making transparent, and sealing. Phosphate-buffered saline was used instead of antibody as the negative control, and breast biopsy was adopted as a positive control. 
The relationship between survivin, Bcl-2, and KAl1 expression levels and cervical cancer invasion metastasis was analyzed.

\section{Analysis of positive expression}

The slices were reviewed by two pathologists using the double blind method. Cells that expressed survivin, Bcl-2, or KAl1 appeared as pale yellow or tan particles. The positive cell number and cellular staining intensity were observed under high magnification (400X) for semi-quantitative analysis. Staining intensity was scored as follows: 0 represented no coloring, 1 represented dyeing to pale yellow, 2 represented dyeing to yellow, and 3 represented dyeing to tan. Five visions of each section were randomly selected for observation and the percentage of positive cells in 500 cells was evaluated for scoring: 0 represented less than 10\% positive cells, 1 represented 10-40\%, 2 represented $40-70 \%$, and 3 represented $70 \%$ or higher. The two scores were added together to provide a measure of positive expression: positive (+), $\geq 2$; negative (-), 0-1.

\section{Statistical analysis}

All statistical analyses were performed using SPSS18.0 software (Chicago, IL). The Pearson $\mathrm{X}^{2}$ test and the Fisher's exact $t$-test were used to assess the differences between the enumeration data. Spearman rank correlation analyses were performed for correlation. $P$ values of less than 0.05 were considered significant.

\section{RESULTS}

\section{Bcl-2, survivin, and KAl1 expression levels in different cervical tissues}

Table 3 shows that the expression levels of survivin and Bcl-2 in cervical cancer tissue (66.7 and $68.9 \%$, respectively) were significantly higher than in normal cervical tissue $(0.0$ and $4.0 \%$, respectively), chronic cervicitis (16.0 and $12.0 \%$, respectively), and CIN (36.7 and $33.3 \%$, respectively) $(P<0.05)$, while KAl1 expression in cervical cancer tissue $(40.0 \%)$ was markedly lower than in normal cervical tissue $(96.0 \%)$, chronic cervicitis $(92.0 \%)$, and CIN $(73.7 \%)(P<$ 0.05). The expression levels of survivin, Bcl-2, and KAI1 in CIN were not statistically different when compared with those for chronic cervicitis tissues, but were statistically different from normal cervical tissue $(P<0.05)$. Survivin and KAl1 expression levels were similar between normal cervical tissue and chronic cervicitis, while Bcl-2 expression was different between normal cervical tissues and chronic cervicitis $(P<0.05)$ (Table 3$)$.

Table 3. Bcl-2, survivin, and KAl1 expression levels $(\mathrm{N}, \%)$ in different cervical tissues.

\begin{tabular}{lcccc}
\hline Group & $\mathrm{N}$ & $\mathrm{Bcl}-2$ & Survivin & KAl1 \\
\hline Normal control & 25 & $1(4.0)^{\#}$ & $0(0)^{\#}$ & $24(96.0)^{\#}$ \\
Chronic cervicitis & 25 & $3(12.0)^{\#}$ & $4(16.0)^{* \#}$ & $23(92.0)^{\#}$ \\
CIN & 30 & $10(33.3)^{\star \#}$ & $11(36.7)^{* \#}$ & $22(73.7)^{* \#}$ \\
Cervical cancer & 45 & $31(68.9)^{*}$ & $30(66.7)^{*}$ & $18(40.0)^{*}$ \\
$X^{2}$ & - & 38.5802 & 36.7766 & 32.9956 \\
P value & - & 0.00 & 0.00 & 0.00 \\
\hline
\end{tabular}

$\overline{\mathrm{CIN}}=$ cervical intraepithelial neoplasia. ${ }^{*} \mathrm{P}<0.05$ compared with normal control; ${ }^{\mathrm{P}}<0.05$ compared with cervical cancer. 
Bcl-2 was mainly expressed in the cytoplasm, while survivin was mainly expressed in the cytoplasm and cell membrane, and their expression levels increased in increments from cervicitis to $\mathrm{CIN}$ and cervical cancer. KAI1 was mainly expressed in the cell membrane, and its expression declined from cervicitis to CIN and cervical cancer (Figure 1).

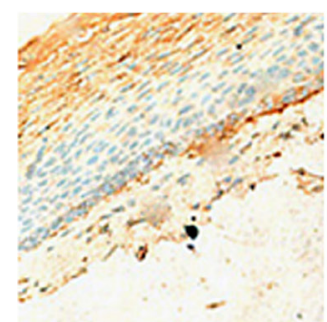

Bcl-2 normal control

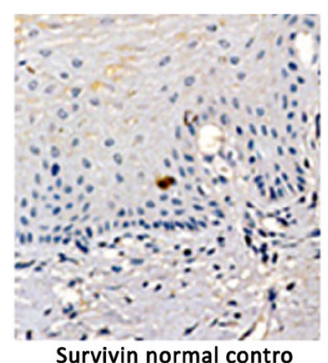

Survivin normal contro

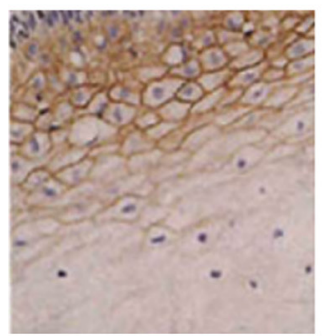

KAI1 normal control

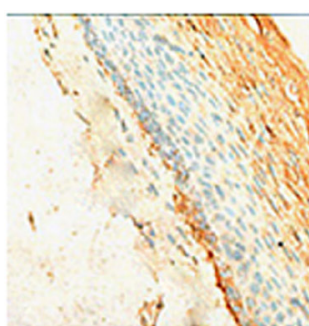

Bcl-2 chronic cervicitis

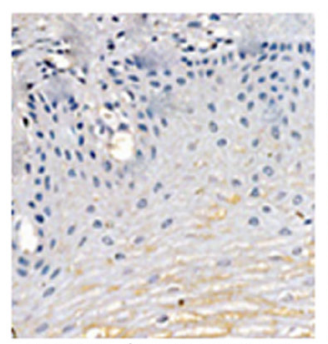

Survivin chronic cervicitis

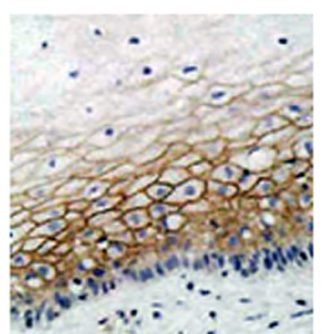

KAI1 chronic cervicitis

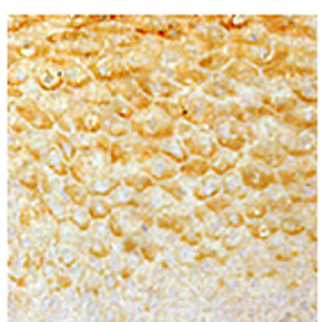

Bcl-2 CIN

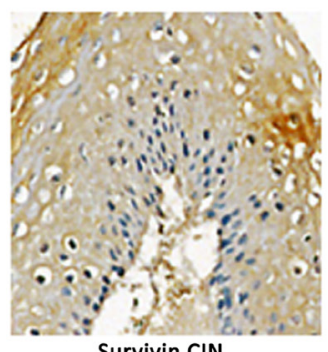

Survivin CIN

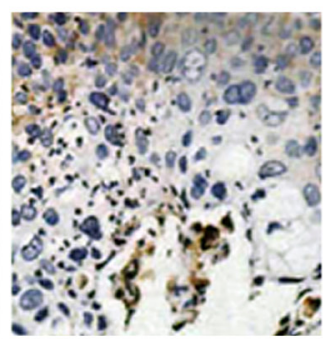

KAI1 CIN

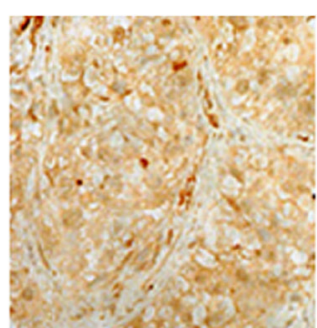

Bcl-2 cervical cancer

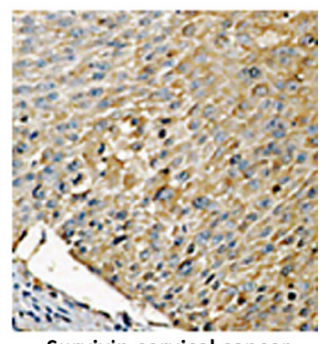

Survivin cervical cance

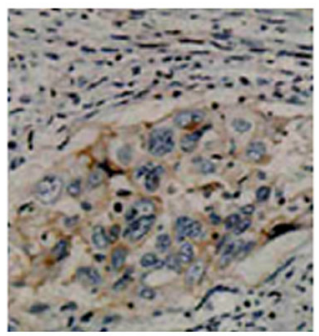

KAl1 cervical cancer

Figure 1. Bcl-2, survivin, and KAl1 expression in different cervical tissues.

\section{Relationship between Bcl-2, survivin, and KAl1 expression levels and clinicopathological characteristics in cervical cancer}

There were no statistically significant differences in the expression levels of the three proteins between young ( $<35$ years) and older ( $\geq 35$ years) patients $(P>0.05)$. Bcl-2 expression was associated with clinical stage $(P<0.05)$, but not with tissue type, tumor type, differentiation, or lymph node metastasis $(P>0.05)$. Survivin expression was positively correlated with clinical stage, and was associated with lymph node metastasis $(P<0.05)$, but not with tumor type, tissue type, or differentiation $(P>0.05)$. KAl1 expression was associated with lymph node metastasis, differentiation, and clinical stage $(P<0.05)$, but not with tumor type or tissue type $(P>0.05)$ (Table 4). 
Table 4. Relationship between Bcl-2, survivin, and KAl1 expression levels and clinicopathological characteristics in cervical cancer $(\mathrm{N}, \%)$.

\begin{tabular}{|c|c|c|c|c|c|c|c|}
\hline Factors & $\mathrm{N}$ & Bcl-2 & $P$ value & Survivin & $P$ value & KAl1 & $P$ value \\
\hline \multicolumn{8}{|l|}{ Age (years) } \\
\hline$<35$ & 20 & $14(70.0)$ & \multirow[t]{2}{*}{0.88} & $14(70.0)$ & \multirow[t]{2}{*}{0.46} & $8(40.0)$ & \multirow[t]{2}{*}{0.75} \\
\hline$\geq 35$ & 25 & $17(68.0)$ & & $16(64.0)$ & & $10(40.0)$ & \\
\hline \multicolumn{8}{|l|}{ Tumor type } \\
\hline Exophytic & 24 & $16(66.7)$ & \multirow[t]{3}{*}{0.91} & $16(66.7)$ & \multirow[t]{3}{*}{0.95} & $9(37.5)$ & \multirow[t]{3}{*}{0.86} \\
\hline Endophytic & 13 & $9(69.2)$ & & $9(69.2)$ & & $6(46.2)$ & \\
\hline Ulcerative & 8 & $6(75.0)$ & & $5(62.5)$ & & $3(37.5)$ & \\
\hline \multicolumn{8}{|l|}{ Tissue type } \\
\hline Squamous cell carcinoma & 41 & $28(68.3)$ & \multirow[t]{2}{*}{0.78} & $28(68.29)$ & \multirow[t]{2}{*}{0.85} & $17(41.4)$ & \multirow[t]{2}{*}{0.91} \\
\hline Adenocarcinoma & 4 & $3(75.0)$ & & $2(50.0)$ & & $1(25.0)$ & \\
\hline \multicolumn{8}{|l|}{ Clinical stage } \\
\hline la & 4 & $1(25.0)$ & \multirow[t]{3}{*}{0.03} & $1(25.0)$ & \multirow[t]{3}{*}{0.04} & $4(100.0)$ & \multirow[t]{3}{*}{0.03} \\
\hline Ib-IIa & 38 & $27(71.1)$ & & $26(68.4)$ & & $13(34.2)$ & \\
\hline IIb-III & 3 & $3(100.0)$ & & $3(100.0)$ & & $1(33.3)$ & \\
\hline \multicolumn{8}{|l|}{ Differentiation } \\
\hline Good & 14 & $9(64.3)$ & \multirow[t]{3}{*}{0.47} & $8(64.2)$ & \multirow[t]{3}{*}{0.35} & $10(71.4)$ & \multirow[t]{3}{*}{0.02} \\
\hline Moderate & 28 & $19(67.9)$ & & $19(67.9)$ & & $8(28.6)$ & \\
\hline Poor & 3 & $3(100.0)$ & & $3(100.0)$ & & $0(0.0)$ & \\
\hline \multicolumn{8}{|l|}{ Lymph node metastasis } \\
\hline With & 29 & $19(65.5)$ & \multirow[t]{2}{*}{0.51} & $24(82.7)$ & \multirow[t]{2}{*}{0.002} & $7(24.1)$ & \multirow[t]{2}{*}{0.01} \\
\hline Without & 16 & $12(75.0)$ & & $6(37.5)$ & & $11(68.75)$ & \\
\hline
\end{tabular}

\section{Correlation analysis of Bcl-2, survivin, and KAl1 expression levels in cervical cancer}

Among the 30 cases of survivin-positive cervical cancer tissue, 26 cases presented positive $\mathrm{Bcl}-2$ expression, and 8 cases presented positive KAl1 expression. Of the 15 cases of survivinnegative cervical cancer tissue, 5 showed positive Bcl-2 expression and 10 showed positive KAl1 expression. Survivin expression was positively correlated with Bcl-2 $(r=0.576, P<0.05)$, but not with KAl1 expression ( $r=0.096, P>0.05)$. In the 31 cases of $\mathrm{Bcl}-2$-positive cervical cancer tissue, 11 cases showed positive KAI1 expression, and in the 14 cases of Bcl-2-negative cervical cancer tissue, 7 cases showed positive KAl1 expression. No significant correlation between Bcl-2 and KAl1 was observed $(r=0.094, P>0.05)$.

\section{DISCUSSION}

The pathological process of cervical cancer is related to tumor-suppressor gene inactivation and proto-oncogene activation. Apoptosis is one of the basic functions of the cell. A dynamic balance is maintained between apoptosis and proliferation under normal conditions. Apoptosis disruption may lead to many diseases. The BCL2 gene belongs to the anti-apoptotic genes in the BCL2 gene family and a translocation between chromosome 14 and 18 results in the overexpression of the BCL2 gene. As a membrane protein, $\mathrm{Bcl}-2$ is overexpressed in cervical cancer tissue. It can prevent apoptosis and prolong the cell life cycle by inhibiting $\mathrm{Ca}^{2+}$ increases in the cell, closing cell nuclear transportation, and acting as an antioxidant (Saitoh et al., 1999; Eijsink et al., 2010). In this study, the expression rate of Bcl-2 in cervical cancer was significantly higher than in the normal cervix, chronic cervicitis, or CIN tissues. Bcl-2 protein, mainly located in the rough endoplasmic reticulum and the mitochondrial membrane, can enhance resistance to apoptosis factors. Bcl-2 overexpression can inhibit cell apoptosis, and cervical cancer can develop under the regulation of growth suppressor and proliferation genes (Han et al., 2012). Bcl-2 is expressed in normal 
epithelial tissue. This study showed that Bcl-2 expression increased with an increase in the degree of cervical tissue lesions. Bcl-2 expression was positively correlated with clinical stage, but not with pathologic stage or lymph node metastasis. Following the increase of cervical cancer clinical stage, the significant elevation in the expression level of Bcl-2 suggested that it is closely related to cervical cancer development, and its expression is associated with cervical cancer infiltration (Lu et al., 2012). The anti-apoptotic mechanism of Bcl-2 is related to mitochondrial redox state changes, cell membrane potential control, the apoptosis protein precursor APAF-1, cytochrome $\mathrm{C}$ release, caspase-9 activation, and blocking of the caspase pathway (Wu et al., 2012). It has been suggested that Bcl-2 affects the early stages of cervical cancer, while its effect is not significant in the late stages. Cervical tissue cancerization may continue to activate other disease-causing genes, which could inhibit $\mathrm{Bcl}-2$ expression to a certain extent. The pathological process of cervical lesions is a result of multiple gene interactions (Manusirivithaya et al., 2006; Wu et al., 2012). Bcl2 is an important protein in cervical cancer cell apoptosis, and it can regulate cell apoptosis and proliferation (Manusirivithaya et al., 2006). Multiple studies have reported that the 5 -year survival rate in cervical cancer patients is positively correlated with $\mathrm{Bcl}-2$ expression, which may suggest that $\mathrm{Bcl}-2$ controls cell apoptosis but fails to promote cell proliferation. Researchers have not found a correlation between $\mathrm{Bcl}-2$ expression and the prognosis of patients with recurrent cervical cancer (Xue et al., 2011; Guo and Li, 2012). The BIRC5 gene, which encodes the protein survivin and has the locus $17 \mathrm{q} 25$, can influence the apoptosis pathway (by acting on caspases and promoting cell division). Survivin may inhibit apoptosis through two pathways: promoting CDK4 (cyclin-dependent kinases) and releasing p21; and inhibiting caspase-7 and caspase-3 activity (Sukpan et al., 2011; Kogo et al., 2015). Survivin distribution is different from that of Bcl-2 and shows strong tissue selectivity. It is not expressed in most healthy tissues but is highly expressed in fetal tissues. In addition to the gonads and thymus, it is overexpressed in most tumor tissues, such as cancerous lung, colon, and cervical tissues. Survivin can also be expressed in precancerous tissue. Our study results showed that survivin expression increased following an increase in the degree of cervical lesions. Survivin expression was associated with clinical stage and lymph node metastasis; its expression level increased with the increase in clinical stage. Survivin expression increased with a decrease in the degree of cervical cancer tissue differentiation. Survivin overexpression can promote cervical squamous carcinoma. Patients with lymph node metastasis present significantly higher survivin expression than patients without lymphatic metastasis. This suggests that survivin plays a role in inhibiting apoptosis and promoting proliferation in cervical cancer development, which might be reflected in tumor invasiveness and poor prognosis. No difference was found in survivin expression between cervical adenocarcinoma and squamous carcinoma in this study. This may have been due to the small sample size of the collected cervical adenocarcinoma. The question of whether survivin can predict cervical cancer prognosis requires further investigated owing to the lack of survival time follow-up data.

The CD82 gene, which encodes the KAl1 protein, is a tumor metastasis suppressor gene and has the chromosomal locus 11p11.2. KAl1 is mainly located on the cell membrane and is downregulated in colon, pancreatic, and cervical cancers. It can inhibit tumor metastasis without affecting tumor formation. Its expression in cervical cancer tissue is reduced or even absent, and its relationship with clinical features has not yet been definitively determined. It is thought that a decrease in KAl1 expression is associated with the early stages of cervical cancer (Xiong et al., 2005; Liu et al., 2014). In our research, KAl1 expression levels in cervical cancer tissue decreased with the increasing degree of cervical tissue pathological changes. KAl1 expression was associated 
with lymph node metastasis, differentiation, and clinical stage. This suggests that KAl1 expression was downregulated with cervical lesion development and tissue differentiation decrease, as its expression in poorly differentiated cervical cancer tissue was significantly lower than in highly differentiated tissue. KAI1 expression was downregulated in the late clinical stages. The patient's prognosis worsens with increase in clinical stage. This suggests that KAl1 decline might be an early event in the process of cervical cancer. The level of KAl1 protein may be related to patient prognosis. However, the relationship between the level of KAI1 and invasion and metastasis in cervical cancer remains controversial (Schindl et al., 2001; Liu et al., 2003; Xiong et al., 2005). Our results showed that KAl1 expression was associated with lymph node metastasis. The KAI1 protein participates in reactions between cells or between the extracellular matrix and cells, and affects cancer cell invasion and metastasis. The downregulated expression of KAI1 is related to cervical cancer prognosis. Tumor metastasis is the main cause of death in the majority of malignant tumor patients. Our study suggested that patients with downregulated KAl1 expression, late clinical stage, worse tissue type, and the presence of lymph node metastasis might havea higher risk of recurrence. Lymph node metastasis, clinical stage, and KAl1 protein expression were relative prognosis factors for cervical cancer patients.

Correlation analyses of survivin, Bcl-2, and KAl1 expression levels in 45 cases of cervical cancer were performed. Survivin expression was positively correlated with Bcl-2 ( $r=0.576, \mathrm{P}<$ $0.05)$, which may be related to BIRC5 gene chromosome instability. The loci of the BIRC5 gene are involved in $B C L 2$ gene locus $t(14,18)$ translocation and $B C L 2$ gene activation. In addition, BIRC5 and $B C L 2$ genes are regulated by similar promoter sequences, which suggests that they may have similar mechanisms for regulating cell transcription activity (Schindl et al., 2001). The BCL2 gene regulates apoptosis by preventing cytochrome $C$ release by mitochondria, which is upstream in the caspase cascade. BIRC5 may block the apoptosis pathway (by affecting caspases and promoting the cell division pathway). Two anti-apoptotic pathways may work together in cervical cancer. Survivin and Bcl-2 both showed no significant correlation with KAl1 expression $(r=0.096$, $\mathrm{P}>0.05 ; \mathrm{r}=0.094, \mathrm{P}>0.05$, respectively). BIRC5 and BCL2 are both anti-apoptotic genes, and they participate in cervical cancer lesions through regulating cell apoptosis. $C D 82$ is a tumor metastasis suppressor gene and works by interacting with CD51 and CD9 (Miranti, 2009). To summarize, survivin, $\mathrm{Bcl}-2$, and KAl1 are expressed abnormally in cervical cancer tissue, and their expression levels have a certain correlation with cervical cancer occurrence and development. KAl1 expression is negatively correlated with cervical cancer metastasis. Survivin, Bcl-2, and KAl1 are cervical cancer recurrence- and prognosis-related factors, although their role in cervical cancer prognosis requires confirmation.

\section{Conflicts of interest}

The authors declare no conflict of interest.

\section{REFERENCES}

Adefolaju GA, Theron KE and Hosie MJ (2014). Effects of HIV protease, nucleoside/non-nucleoside reverse transcriptase inhibitors on Bax, Bcl-2 and apoptosis in two cervical cell lines. Biomed. Pharmacother. 68: 241-251.

Eijsink JJ, Noordhuis MG, ten Hoor KA, Kok M, et al. (2010). The epidermal growth factor receptor pathway in relation to pelvic lymph node metastasis and survival in early-stage cervical cancer. Hum. Pathol. 41: 1735-1741.

Guo Q and Li Y (2012). Analysis on expression of survivin and PTEN in vocal cords polyps, papilloma of larynx and laryngeal squamous cell carcinoma. Lin Chung Er Bi Yan Hou Tou Jing Wai Ke Za Zhi 26: 704-707. 
Han S, Li L, Jia X, Ou W, et al. (2012). A molecular beacon-based method for screening cervical cancer. J. Nanosci. Nanotechnol. 12: 8282-8286.

Kogo R, How C, Chaudary N, Bruce J, et al. (2015). The microRNA-218 Survivin axis regulates migration, invasion, and lymph node metastasis in cervical cancer. Oncotarget 6: 1090-1100.

Liu FS, Dong JT, Chen JT, Hsieh YT, et al. (2003). KAI1 metastasis suppressor protein is down-regulated during the progression of human endometrial cancer. Clin. Cancer Res. 9: 1393-1398.

Liu HN, Shi HR, Zhao XL, Zhang RT, et al. (2014). The TLR3, PI3K, survivin, FasL, and Fas genes as major risk factors of occurrence and development of cervical cancer disease. Gene 550: 27-32.

Lu D, Qian J, Yin X, Xiao Q, et al. (2012). Expression of PTEN and survivin in cervical cancer: promising biological markers for early diagnosis and prognostic evaluation. Br. J. Biomed. Sci. 69: 143-146.

Manusirivithaya S, Siriaunkgul S, Khunamornpong S, Sripramote M, et al. (2006). Association between Bcl-2 expression and tumor recurrence in cervical cancer: a matched case-control study. Gynecol. Oncol. 102: 263-269.

Miranti CK (2009). Controlling cell surface dynamics and signaling: how CD82/KAI1 suppresses metastasis. Cell. Signal. 21: 196-211.

Ouyang YW, Pan XL, Peng ZL, Qu Y, et al. (2008a). Expression of metastasis suppressor gene KAl1 in cervical carcinoma and infections of HPV16 E6, E7 and HPV18 E6/E7. Sichuan Da Xue Xue Bao Yi Xue Ban 39: 410-413.

Ouyang YW, Pan XL, Qu Y, Peng ZL, et al. (2008b). Effect of metastasis suppressor gene KAl1 on the proliferation and invasive ability of cervical carcinoma cells. Sichuan Da Xue Xue Bao Yi Xue Ban 39: 753-756.

Ozden S, Tiber P, Ozgen Z, Ozyurt H, et al. (2014). Expression of TRF2 and its prognostic relevance in advanced stage cervical cancer patients. Biol. Res. 47: 61.

Saitoh Y, Yaginuma Y and Ishikawa M (1999). Analysis of Bcl-2, Bax and Survivin genes in uterine cancer. Int. J. Oncol. 15: 137-141.

SchindI M, Bachtiary B, Dreier B, Birner P, et al. (2001). Impact of human papillomavirus infection on the expression of the KAI1 metastasis suppressor protein in invasive cervical cancer. Cancer Lett. 162: 261-266.

Singh A, Sharma H, Salhan S, Gupta SD, et al. (2004). Evaluation of expression of apoptosis-related proteins and their correlation with HPV, telomerase activity, and apoptotic index in cervical cancer. Pathobiology 71: 314-322.

Sukpan K, Settakorn J, Khunamornpong S, Cheewakriangkrai C, et al. (2011). Expression of survivin, CD117, and C-erbB-2 in neuroendocrine carcinoma of the uterine cervix. IInt. J. Gynecol. Cancer 21: 911-917.

Wang M, Wang B and Wang X (2001). A novel antiapoptosis gene, survivin, bcl-2, p53 expression in cervical carcinomas. Zhonghua Fu Chan Ke Za Zhi 36: 546-548.

Winer I, Alvarado-Cabrero I, Hassan O, Ahmed QF, et al. (2015). The prognostic significance of histologic type in early stage cervical cancer - A multi-institutional study. Gynecol. Oncol. 137: 474-478.

Wu SF, Zhang JW, Qian WY, Yang YB, et al. (2012). Altered expression of survivin, Fas and FasL contributed to cervical cancer development and metastasis. Eur. Rev. Med. Pharmacol. Sci. 16: 2044-2050.

Xiong Y, Liang LZ, Yan XJ, Yuan SH, et al. (2005). Expression of metastasis suppressor gene KAI1/CD82 in cervical squamous cell carcinoma and its clinical significance. Ai Zheng 24: 110-115.

Xue Y, An R, Zhang D, Zhao J, et al. (2011). Detection of survivin expression in cervical cancer cells using molecular beacon imaging: new strategy for the diagnosis of cervical cancer. Eur. J. Obstet. Gynecol. Reprod. Biol. 159: 204-208. 